\title{
Analytical simulation of magneto-hemodynamic flow in a semi-porous channel using the Polynomial Least Squares Method
}

\author{
Bogdan Căruntu ${ }^{1, *}$, Constantin Bota ${ }^{1, * *}$, and Olivia Bundău ${ }^{1, * * *}$ \\ 1 "Politehnica" University of Timişoara Dept. of Mathematics, P-ta Victoriei 2, Timişoara, Romania
}

\begin{abstract}
The present article proposes a new analytical approximate solution for the magneto-hemodynamic laminar viscous flow of a conducting physiological fluid in a semi-porous channel under a transverse magnetic field, solution obtained by using the Polynomial Least Squares Method (PLSM). A comparison of our approximate solutions obtained by PLSM with previously computed approximate solutions illustrates the accuracy of our method. A discussion of the effects of the parameters $\mathrm{Re}$ (the Reynolds number) and $\mathrm{Ha}$ (the Hartmann number) on the blood flow velocity is included.
\end{abstract}

\section{Introduction}

The study of the properties of the blood flow through the vessels is very important due to the fact that the modern diagnose and treatment of cardiovascular diseases relies on the theoretical analysis of physical phenomena of blood flow.

According to numerous analogies that exist between the functioning of the heart and a pump, the study of blood flow can be achieved using mechanical models and the blood flow can be likened to the flow of fluid through a network of tubes. Consequently, the mechanics of the heart and the flow of blood through the elastic vessels are studied using hydrodynamics, which deals with the study of the physical phenomena of circulation. Various models have been developed for the study of the hydrodynamic flow of blood through the vessels. For example, in [1] the authors studied the blood flow in small diameter tubes while in [2] the blood flow in small curved tubes is modeled.

The blood flow may be influenced by a magnetic field because the composition of the red blood cells includes biomagnetic substances. The effect of a magnetic field on the blood flow has been analyzed theoretically by treating blood as an electrically conductive fluid. Assuming blood as a magnetic fluid, it may be possible to control blood pressure and its flow behaviour by using an appropriate magnetic field. In [3], the authors proposed a mathematical model of the blood flow in small blood vessel in the presence of a magnetic field. Also, in [4], the authors analyzed the mechanism of interaction between the red blood cells and an external magnetic field, showing that a magnetic field can modulate the blood flow. Other interesting studies on the magnetic properties of the blood include [5], [6], [7], [8], [9].

\footnotetext{
*e-mail: bogdan.caruntu@upt.ro

**e-mail: constantin.bota@upt.ro

***e-mail: olivia.bundau@upt.ro
} 
There are of course many mathematical models which describe parts of the human circulatory system (for example [10], [11], [12], [13]), usually the blood flow being modeled by using differential equations, mostly nonlinear ones. Unfortunately, finding exact solutions for such equations is possible only in some particular cases. This justifies the need to resort to approximate methods for calculating approximate solutions, since the approximate solutions could provide important information about the phenomena studied.

In the present paper, based on the study from [5], we present a new method for the computation of approximate solutions for the transformed coupled ordinary nonlinear differential equations modelling the laminar magneto-hemodynamic viscous flow in a semi-porous channel.

\section{The mathematical model}

The laminar two-dimensional stationary flow of an incompressible electrically-conducting viscous fluid in a semi-porous channel is considered. The channel is composed of a long rectangular plate of length $L_{x}{ }^{*}$ in uniform translation in $x^{*}$ direction and an infinite porous plate. The distance between the two plates is $h$ and an uniform magnetic field $B$ is imposed in the $y^{*}$ direction. The axial and normal velocity components are defined as $u^{*}$ and $v^{*}$, and are parallel to the axial normal coordinates $x^{*}$ and $y^{*}$, respectively. Also, a normal transpiration velocity, $q$, is present at the porous wall.

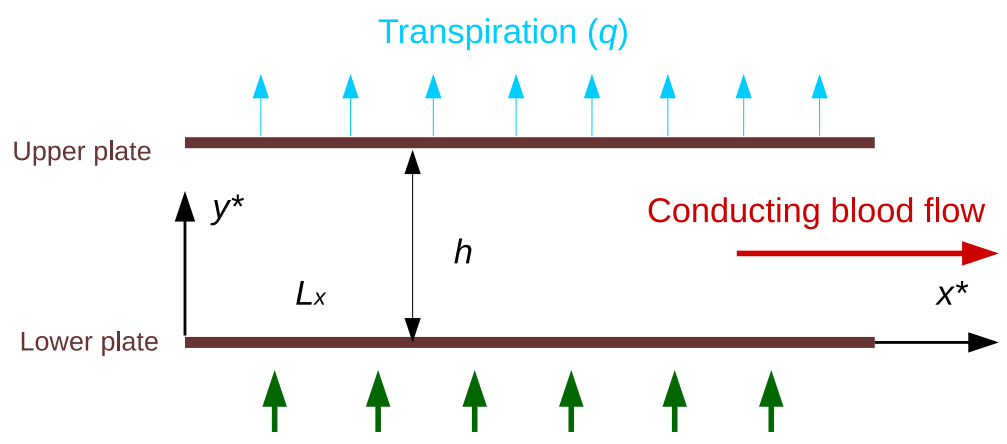

Axial uniform static magnetic field $(B)$

Figure 1: Visualization of the problem

According to [5], the fluid flow in a semi-porous channel is described by the following equations:

$$
\begin{gathered}
\frac{\partial u^{*}}{\partial x^{*}}+\frac{\partial v^{*}}{\partial y^{*}}=0 \\
u^{*} \frac{\partial u^{*}}{\partial x^{*}}+v^{*} \frac{\partial u^{*}}{\partial y^{*}}=-\frac{1}{\rho} \frac{\partial P^{*}}{\partial x^{*}}+v\left(\frac{\partial^{2} u^{*}}{\partial x^{* 2}}+\frac{\partial^{2} u^{*}}{\partial y^{* 2}}\right)-u^{*} \frac{\sigma B^{2}}{\rho} \\
u^{*} \frac{\partial v^{*}}{\partial x^{*}}+v^{*} \frac{\partial v^{*}}{\partial y^{*}}=-\frac{1}{\rho} \frac{\partial P^{*}}{\partial y^{*}}+v\left(\frac{\partial^{2} v^{*}}{\partial x^{* 2}}+\frac{\partial^{2} v^{*}}{\partial y^{* 2}}\right)
\end{gathered}
$$

where $\rho, P^{*}$ and $v$ are density, pressure and dynamic viscosity, respectively. 
The boundary conditions are

$$
y^{*}=0: u^{*}=u_{0}^{*}, v^{*}=0
$$

and

$$
y^{*}=h: u^{*}=0, v^{*}=-q .
$$

The mean velocity $U$ is evaluated using the following relation:

$$
U h=\int_{0}^{h} u^{*} d y^{*}=L_{x} q
$$

Using the following transformations:

$$
x=\frac{x^{*}}{L_{x}}, y=\frac{y^{*}}{h}, u=\frac{u^{*}}{U}, v=\frac{v^{*}}{q}, P_{y}=\frac{P^{*}}{\rho q^{2}} .
$$

into (1)-(3), we obtain the following equations

$$
\begin{gathered}
\frac{\partial u}{\partial x}+\frac{\partial v}{\partial y}=0 \\
u \frac{\partial u}{\partial x}+v \frac{\partial u}{\partial y}=-\varepsilon^{2} \frac{\partial P_{y}}{\partial x}+\frac{v}{h q}\left(\varepsilon^{2} \frac{\partial^{2} u}{\partial x^{2}}+\frac{\partial^{2} u}{\partial y^{2}}\right)-u \frac{H a^{2}}{R e} \\
u \frac{\partial v}{\partial x}+v \frac{\partial v}{\partial y}=-\frac{\partial P_{y}}{\partial y}+\frac{v}{h q}\left(\varepsilon^{2} \frac{\partial^{2} v}{\partial x^{2}}+\frac{\partial^{2} v}{\partial y^{2}}\right)
\end{gathered}
$$

where the Hartman number is $H a=B h \sqrt{\frac{\sigma}{\rho v}}$ and $R e=\frac{h q}{v}$ is the Reynolds number.

The quantity $\varepsilon$ is the ratio of the distance $h$ and a characteristic length $L_{x}$ of the slider. Using the Berman's similarity transformation from [14], the ratio $\varepsilon$ is eliminated from the equations (6)-(7).

$$
v=-V(y), u=\frac{u^{*}}{U}=u_{0}, U(y)+x \frac{d V}{d y} .
$$

Using the relations (8), we obtain that the quantity $\frac{\partial^{2} P_{y}}{\partial x^{2}}$ is independent of $x$.

A separation of variables leads to:

$$
\begin{gathered}
V^{\prime 2}-V V^{\prime \prime}-\frac{1}{R e} V^{\prime \prime \prime}+\frac{H a^{2}}{R e} V^{\prime}=\varepsilon^{2} \frac{\partial^{2} P_{y}}{\partial x^{2}}=\varepsilon^{2} \frac{1}{x} \frac{\partial P_{y}}{\partial x} \\
U V^{\prime}-V U^{\prime}=\frac{1}{R e}\left(U^{\prime \prime}-H a^{2} U\right) .
\end{gathered}
$$

The right-hand side of (9) is constant. Therefore we derive this equation with respect to $y$, we obtain:

$$
V^{I V}=H a^{2} V^{\prime \prime}+\operatorname{Re}\left(V^{\prime} V^{\prime \prime}-V V^{\prime \prime \prime}\right)
$$

The boundary conditions become:

$$
\begin{gathered}
y=0: U=1, V=0, V^{\prime}=0 \\
y=1: U=0, V=1, V^{\prime}=0
\end{gathered}
$$




\section{The Polynomial Least Squares Method}

For the problem (10-12) we consider the operators:

$$
\begin{aligned}
& D_{1}(V, U)=V^{I V}-H a^{2} V^{\prime \prime}-\operatorname{Re}\left(V^{\prime} V^{\prime \prime}-V V^{\prime \prime \prime}\right) \\
& D_{2}(V, U)=U V^{\prime}-V U^{\prime}-\frac{1}{\operatorname{Re}}\left(U^{\prime \prime}-H a^{2} U\right)
\end{aligned}
$$

We will find approximate polynomial solutions $\tilde{u}=(\tilde{V}, \tilde{U})$ of $(10-12)$ on the $[0,1]$ interval, solutions which satisfy the following conditions:

$$
\begin{gathered}
\left|D_{j}(\tilde{u}(y))\right|<\epsilon, \quad j=1,2 \quad \epsilon>0 \\
U(0)=1, V(0)=0, V^{\prime}(0)=0, \\
U(1)=0, V(1)=1, V^{\prime}(1)=0
\end{gathered}
$$

Definition 1 We call an $\epsilon$-approximate polynomial solution of the system (10)(12) an approximate polynomial solution $\tilde{u}$ satisfying the relations $(15,16)$.

Definition 2 We call a weak $\delta$-approximate polynomial solution of the system (10)-(12) an approximate polynomial solution $\tilde{u}$ satisfying the relations:

$$
\int_{0}^{1} D_{j}^{2}(\tilde{u}(y)) d y \leq \delta, \quad j=1,2
$$

together with the initial conditions (16).

Definition 3 We consider the sequence of polynomials $P_{m}^{j}(y)=a_{0}^{j}+a_{1}^{j} y+\ldots+$ $a_{m}^{j} y^{m}, a_{i}^{j} \in \mathbb{R}, i=0,1, \ldots, m, j=1,2$ satisfying the conditions:

$$
\begin{array}{r}
P_{m}^{1}(0)=0, \quad P_{m}^{2}(0)=1, \quad P_{m}^{1}(1)=0, \quad P_{m}^{2}(1)=1, \\
m>2, \quad m \in \mathbb{N}
\end{array}
$$

We call the sequence of polynomials $P_{m}^{j}(y)$ convergent to the solution of the system (10)-(12) if $\lim _{m \rightarrow \infty} D_{j}\left(P_{m}^{1}(y), P_{m}^{2}(y)\right)=0, \quad j=1,2$.

We will find a weak $\epsilon$-polynomial solutions of the type:

$$
\tilde{u}^{j}(y)=\sum_{k=0}^{m} c_{k}^{j} \cdot y^{k}, \quad m>n, \quad j=1,2 .
$$

where the constants $c_{0}^{j}, c_{1}^{j}, \ldots, c_{m}^{j}, \quad j=1,2$ are calculated using the steps outlined in the following.

- We attach to the system (10)-(12) the following real functional:

$$
J\left(c_{2}^{1}, \ldots, c_{m}^{1}, c_{2}^{2}, \ldots, c_{m}^{2}\right)=\sum_{j=1}^{2} \int_{0}^{1} D_{j}^{2}(\tilde{u}(y)) d y
$$

where $c_{0}^{1}, c_{1}^{1}, c_{0}^{2}, c_{1}^{2}$, are computed as functions of $c_{2}^{1}, \ldots, c_{m}^{1}, c_{2}^{2}, \ldots, c_{m}^{2}$ by using the initial conditions (16). 
- We compute the values of $\bar{c}_{2}^{1}, \ldots, \bar{c}_{m}^{1}, \bar{c}_{2}^{2}, \ldots, \bar{c}_{m}^{2}$ as the values which give the minimum of the functional (18) and the values of $\bar{c}_{0}^{1}, \bar{c}_{1}^{1}, \bar{c}_{0}^{2}, \bar{c}_{1}^{2}$ again as functions of $\bar{c}_{2}^{1}, \ldots, \bar{c}_{m}^{1}, \bar{c}_{2}^{2}, \ldots, \bar{c}_{m}^{2}$ by using the initial conditions.

- Using the constants $\bar{c}_{0}^{1}, \bar{c}_{1}^{1}, \ldots, \bar{c}_{m}^{1}, \bar{c}_{0}^{2}, \bar{c}_{1}^{2}, \ldots, \bar{c}_{m}^{2}$ thus determined, we consider the polynomials:

$$
T_{m}^{j}(y)=\sum_{k=0}^{m} \bar{c}_{k}^{j} \cdot y^{k}, \quad m>2, \quad j=1,2
$$

The following convergence theorem holds:

Theorem 1 The necessary condition for the problem (10)-(12) to admit sequences of polynomials $P_{m}^{j}(y)$ convergent to the solution of this problem is:

$$
\lim _{m \rightarrow \infty} \int_{0}^{1} D_{j}^{2}\left(y, T_{m}^{j}(y)\right) d y=0, \quad j=1,2
$$

Moreover, $\forall \epsilon>0, \exists m_{0} \in \mathbb{N}$ such that $\forall m \in \mathbb{N}, m>m_{0}$ it follows that $T_{m}^{j}(y), \quad j=1,2$ are weak $\epsilon$-approximate polynomial solutions of the system (10)-(12).

Proof. Based on the way the coefficients of the polynomials $T_{m}^{j}(y)$ are computed and taking into account the relations (17)-(19), the following inequality holds:

$$
\begin{array}{r}
0 \leq \int_{0}^{1} D_{j}^{2}\left(t, T_{m}^{j}(y)\right) d y \leq \int_{0}^{1} \sum_{j=1}^{2} D_{j}^{2}\left(y, P_{m}^{j}(y)\right) d y, \\
\forall m \in \mathbb{N}, \quad j=1,2 .
\end{array}
$$

It follows that:

$$
\begin{array}{r}
0 \leq \lim _{m \rightarrow \infty} \int_{0}^{1} D_{j}^{2}\left(t, T_{m}^{j}(y)\right) d t \leq \lim _{m \rightarrow \infty} \int_{0}^{1} \sum_{j=1}^{n} D_{j}^{2}\left(t, P_{m}^{j}(y)\right) d y=0, \\
\forall m \in \mathbb{N}, \quad j=1,2 .
\end{array}
$$

We obtain:

$$
\lim _{m \rightarrow \infty} \int_{0}^{1} D_{j}^{2}\left(t, T_{m}^{j}(y)\right) d y=0, \quad j=1,2 .
$$

From this limit we obtain that $\forall \epsilon>0, \exists m_{0} \in \mathbb{N}$ such that $\forall m \in \mathbb{N}, m>m_{0}$ it follows that $T_{m}^{j}(y)$ is a weak $\epsilon$-approximate polynomial solution of the system (10)-(12), $j=1,2$ q.e.d.

Remark 1 Any $\epsilon$-approximate polynomial solution of the system (10)-(12) is also a weak $\epsilon^{2}$-approximate polynomial solution, but the opposite is not always true. It follows that the set of weak approximate solutions of the system (10)-(12) also contains the approximate solutions of the system.

Taking into account the above remark, in order to find $\epsilon$-approximate polynomial solutions of the system (10-12) by PLSM we will first determine weak approximate polynomial solutions, $\tilde{u}$. If $\left|D_{j}(y, \tilde{u})\right|<\epsilon, j=1,2$ then $\tilde{u}$ is also an $\epsilon$-approximate polynomial solution of the system. 


\section{Numerical simulation}

In this section, applying our method presented in the previous section, we calculate approximate polynomial solutions of the equations (10)-(12) for two cases with particular significant values of $\mathrm{Ha}$ and $\mathrm{Re}$ in order to compare our solutions with those obtained in [5].

\subsection{The case $R e=1$ and $H a=0$}

The case $R e=1$ and $H a=0$ corresponds to a non-conducting blood flow with suction at the channel upper plate. In [5], by using the Homotopy Analysis Method HAM and the Differential Transform Method DTM, Basiri Parsa et al. computed some approximate solutions of the system (10)-(12).

Using the Polynomial Least Squares Method PLSM presented in the previous section we computed several pairs of polynomial approximate solutions for the velocity components, $\mathrm{U}(\mathrm{y})$ and $\mathrm{V}(\mathrm{y})$, of which we present for comparison the following pair, consisting of a fourth degree polynomial approximate solution for the velocity component $\mathrm{U}(\mathrm{y})$ and an eight order polynomial approximate solution for the velocity component $\mathrm{V}(\mathrm{y})$ :

$\tilde{U}_{4}(y)=1-1.4063832055669505 \cdot y+0.13200050161621407 \cdot y^{2}+0.5335999325194117$. $y^{3}-0.25921722856867535 \cdot y^{4}$

$\tilde{V}_{8}(y)=3.227125848989447 \cdot y^{2}-2.3943002960544044 \cdot y^{3}-0.0013709234316412896$. $y^{4}+0.35539804051025803 \cdot y^{5}-0.27631017691788934 \cdot y^{6}+0.10065631236690901 \cdot y^{7}-$ $0.011198805462679129 \cdot y^{8}$

Next we compare the our approximate solutions of the system (10)-(12) computed by PLSM with the previous approximate solutions computed in [5] using HAM and DTM by means of Figures 2 and 3 and Table 1. Since the explicit expressions of the HAM approximations were not given in [5], the figures contains only the comparisons with the DTM approximations, while the comparison with the HAM approximations is presented in the table.

It is possible to compute the absolute error corresponding to an approximate solution as the difference in absolute value between the approximate solution and the numerical solution (in this case computed by using the Wolfram Mathematica software). Figures 2 and 3 present the comparison between the absolute errors corresponding to the approximate solutions from [5] obtained by DTM and the absolute errors corresponding to our approximate solutions computed by PLSM.

The errors corresponding to our PLSM approximate solutions are much smaller than the ones obtained by DTM. Moreover, not only our approximate solutions are more precise, but, at the same time, their expressions are much simpler: fourth/fifth degree polynomials vs. the twenty fourth degree polynomial in [5] in Figure 2, and eighth degree polynomial vs. the eighteenth degree polynomial in [5] in Figure 3.

The comparison is further illustrated by Table 1, which also includes the results obtained in [5] by means of the HAM. The comparison with the approximate results obtained by HAM lead to the same conclusion: the approximate solutions obtained by PLSM are more precise and, at the same time, they present a simpler form.

\subsection{The case $R e=1$ and $H a=1$}

Another case discussed in [5] is the case $R e=1$ and $H a=1$. Under the condition $H a=1$ both Lorentzian hydromagnetic drag and viscous force in the flow are of equal magnitude and the flow is weakly magnetic. 


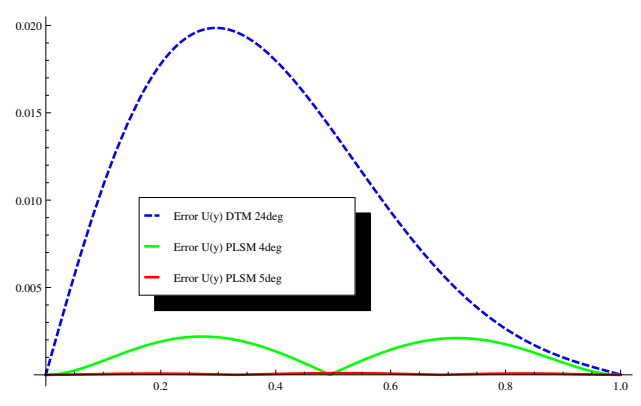

Figure 2: Comparison of absolute errors for the case $R e=1$ and $H a=0$ corresponding to the fourth degree PLSM polynomial approximation $\tilde{U}_{4}$ (solid green curve), the fifth degree PLSM polynomial approximation $\tilde{U}_{5}$ (solid red curve) and the twenty fourth degree polynomial approximation $U_{D T M}$ from [5] (dashed blue curve)

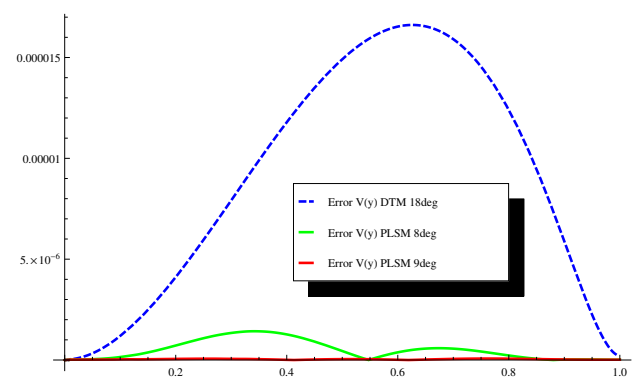

Figure 3: Comparison of absolute errors for the case $R e=1$ and $H a=0$ corresponding to the eighth degree PLSM polynomial approximation $\tilde{V}_{8}$ (solid green curve), the ninth degree PLSM polynomial approximation $\tilde{V}_{9}$ (solid red curve) and the eighteenth degree polynomial approximation $V_{D T M}$ from [5] (dashed blue curve)

Table 1: Comparison of the absolute errors of the approximate solutions for $U$ and $V$ for the case $R e=1$ and $H a=0$.

\begin{tabular}{c|cccccc}
\hline $\mathrm{y}$ & $U_{\text {HAM }}$ & $U_{D T M}$ & $U_{P L S M}$ & $V_{H A M}$ & $V_{D T M}$ & $V_{P L S M}$ \\
& 10 th deg & 24th deg & 5th deg & 10th deg & 18th deg & 8th deg \\
\hline 0.1 & $9.04 \times 10^{-3}$ & $1.08 \times 10^{-2}$ & $4.59 \times 10^{-5}$ & $2.21 \times 10^{-4}$ & $1.19 \times 10^{-6}$ & $1.48 \times 10^{-7}$ \\
0.2 & $1.77 \times 10^{-2}$ & $1.77 \times 10^{-2}$ & $7.09 \times 10^{-5}$ & $5.75 \times 10^{-4}$ & $4.11 \times 10^{-6}$ & $7.17 \times 10^{-7}$ \\
0.3 & $2.71 \times 10^{-2}$ & $1.98 \times 10^{-2}$ & $2.51 \times 10^{-5}$ & $6.47 \times 10^{-4}$ & $7.91 \times 10^{-6}$ & $1.34 \times 10^{-6}$ \\
0.4 & $3.45 \times 10^{-2}$ & $1.79 \times 10^{-2}$ & $5.15 \times 10^{-5}$ & $3.03 \times 10^{-4}$ & $1.17 \times 10^{-5}$ & $1.27 \times 10^{-6}$ \\
0.5 & $3.73 \times 10^{-2}$ & $1.39 \times 10^{-2}$ & $9.50 \times 10^{-5}$ & $2.63 \times 10^{-4}$ & $1.49 \times 10^{-5}$ & $4.45 \times 10^{-7}$ \\
0.6 & $3.46 \times 10^{-2}$ & $9.33 \times 10^{-3}$ & $6.83 \times 10^{-5}$ & $7.02 \times 10^{-4}$ & $1.65 \times 10^{-5}$ & $3.81 \times 10^{-7}$ \\
0.7 & $2.77 \times 10^{-2}$ & $5.39 \times 10^{-3}$ & $9.88 \times 10^{-6}$ & $7.62 \times 10^{-4}$ & $1.59 \times 10^{-5}$ & $5.63 \times 10^{-7}$ \\
0.8 & $1.87 \times 10^{-2}$ & $2.63 \times 10^{-3}$ & $7.22 \times 10^{-5}$ & $4.73 \times 10^{-4}$ & $1.23 \times 10^{-5}$ & $2.24 \times 10^{-7}$ \\
0.9 & $9.27 \times 10^{-3}$ & $9.33 \times 10^{-4}$ & $5.45 \times 10^{-5}$ & $1.25 \times 10^{-4}$ & $5.88 \times 10^{-6}$ & $2.11 \times 10^{-8}$ \\
1 & $4.47 \times 10^{-9}$ & $1.21 \times 10^{-5}$ & $4.47 \times 10^{-9}$ & $1.54 \times 10^{-8}$ & $2.05 \times 10^{-7}$ & $1.54 \times 10^{-8}$ \\
\hline
\end{tabular}


For this case we also computed several pairs of approximate solutions by using PLSM. For example, the pair of polynomial approximate solutions of degree 5 and 8 for the velocity components $\mathrm{U}(\mathrm{y})$ and $\mathrm{V}(\mathrm{y})$, respectively, are:

$$
\tilde{U}_{5}(y)=1-1.6636317583472349 \cdot y+0.5168263132281002 \cdot y^{2}+0.7002202133671248 \text {. }
$$
$y^{3}-0.7958586935242045 \cdot y^{4}+0.24244392527621436 \cdot y^{5}$

$$
\tilde{V}_{8}(y)=3.271178594900408 \cdot y^{2}-2.5917324068340664 \cdot y^{3}+0.2724395060586526 \text {. }
$$
$y^{4}+0.2278634960023271 \cdot y^{5}-0.27403460540765984 \cdot y^{6}+0.10631116334161439 \cdot y^{7}-$ $0.01202574806127546 \cdot y^{8}$

Performing the same comparisons presented in the previous case, Figures 4 and 5 and Table 2 lead to the same conclusions: the approximate solutions obtained by PLSM are more precise even though their expression is much simpler in comparison with previously computed approximate solutions.

We also mention the fact that, as a part of the more complex discussion included in the following section, we also computed approximate solutions corresponding a wide range of values of the parameters $\mathrm{Re}$ and $\mathrm{Ha}$ and, for all the computed solutions, the above conclusions remained true.

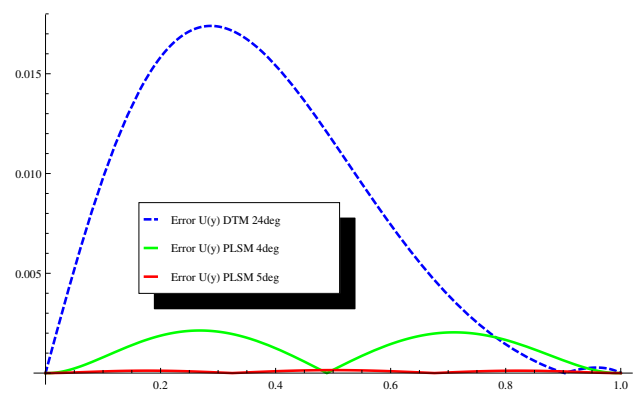

Figure 4: Comparison of absolute errors for the case $R e=1$ and $H a=1$ corresponding to the fourth degree PLSM polynomial approximation $\tilde{U}_{4}$ (solid green curve), the fifth degree PLSM polynomial approximation $\tilde{U}_{5}$ (solid red curve, close to the axis) and the twenty fourth degree polynomial approximation $U_{D T M}$ from [5] (dashed blue curve)

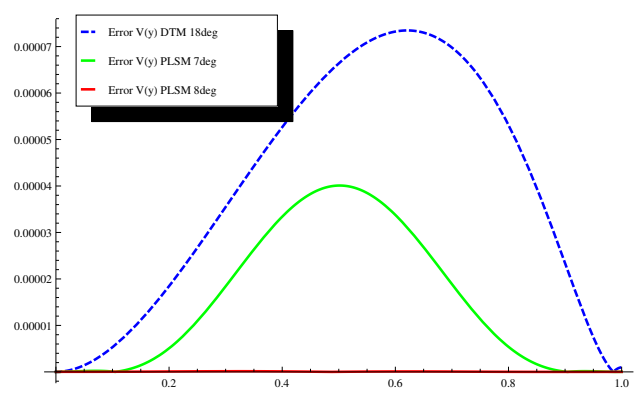

Figure 5: Comparison of absolute errors for the case $R e=1$ and $H a=1$ corresponding to the seventh degree PLSM polynomial approximation $\tilde{V}_{7}$ (solid green curve), the eighth degree PLSM polynomial approximation $\tilde{V}_{8}$ (solid red curve, very close to the axis) and the eighteenth degree polynomial approximation $V_{D T M}$ from [5] (dashed blue curve) 
Table 2: Comparison of the absolute errors of the approximate solutions for $U$ and $V$ for the case $R e=1$ and $H a=1$.

\begin{tabular}{c|cccccc}
\hline $\mathrm{y}$ & $U_{H A M}$ & $U_{D T M}$ & $U_{P L S M}$ & $V_{H A M}$ & $V_{D T M}$ & $V_{P L S M}$ \\
& 10 th deg & 24th deg & 5th deg & 10 th deg & 18 th deg & 8th deg \\
\hline 0.1 & $1.19 \times 10^{-2}$ & $9.78 \times 10^{-3}$ & $8.22 \times 10^{-5}$ & $4.90 \times 10^{-4}$ & $5.31 \times 10^{-6}$ & $3.06 \times 10^{-8}$ \\
0.2 & $1.59 \times 10^{-2}$ & $1.58 \times 10^{-2}$ & $1.17 \times 10^{-4}$ & $1.15 \times 10^{-3}$ & $1.84 \times 10^{-5}$ & $8.27 \times 10^{-8}$ \\
0.3 & $1.25 \times 10^{-2}$ & $1.73 \times 10^{-2}$ & $3.15 \times 10^{-5}$ & $1.19 \times 10^{-3}$ & $3.54 \times 10^{-5}$ & $1.37 \times 10^{-7}$ \\
0.4 & $5.92 \times 10^{-3}$ & $1.54 \times 10^{-2}$ & $9.22 \times 10^{-5}$ & $4.36 \times 10^{-4}$ & $5.26 \times 10^{-5}$ & $1.04 \times 10^{-7}$ \\
0.5 & $1.62 \times 10^{-4}$ & $1.16 \times 10^{-2}$ & $1.48 \times 10^{-4}$ & $7.25 \times 10^{-4}$ & $6.64 \times 10^{-5}$ & $1.52 \times 10^{-8}$ \\
0.6 & $2.79 \times 10^{-3}$ & $7.43 \times 10^{-3}$ & $9.29 \times 10^{-5}$ & $1.66 \times 10^{-3}$ & $7.32 \times 10^{-5}$ & $9.12 \times 10^{-8}$ \\
0.7 & $3.20 \times 10^{-3}$ & $3.90 \times 10^{-3}$ & $3.03 \times 10^{-5}$ & $1.90 \times 10^{-3}$ & $6.97 \times 10^{-5}$ & $6.89 \times 10^{-8}$ \\
0.8 & $2.34 \times 10^{-3}$ & $1.43 \times 10^{-3}$ & $1.16 \times 10^{-4}$ & $1.35 \times 10^{-3}$ & $5.31 \times 10^{-5}$ & $1.55 \times 10^{-8}$ \\
0.9 & $1.21 \times 10^{-3}$ & $2.36 \times 10^{-5}$ & $8.04 \times 10^{-5}$ & $4.73 \times 10^{-4}$ & $2.33 \times 10^{-5}$ & $6.71 \times 10^{-10}$ \\
1 & $7.96 \times 10^{-9}$ & $5.79 \times 10^{-6}$ & $7.96 \times 10^{-9}$ & $9.14 \times 10^{-9}$ & $9.70 \times 10^{-7}$ & $9.15 \times 10^{-9}$ \\
\hline
\end{tabular}

\section{Discussion}

In this section, based on an extensive computation of approximate solutions corresponding to a wide range of values for the Hartmann number $\mathrm{Ha}$ and for the Reynolds number Re, we present our results, which are in close agreement with previous results presented in [5], [15], [16], [17] and other studies.

First, for fixed values of the Hartmann number we studied the influence of the Reynolds number on the velocities $U$ and $V$. For both cases studied in [5], the non-conducting case $H a=0$ and the weakly magnetic flow case $H a=1$, we draw similar conclusions: an increase of the Reynolds number Re leads to a slight increase of the $V(y)$ component of blood velocity and to a significant decrease of the $U(y)$ component. As noted in [5], these results can be attributed to the fact that an increase of $R e$ corresponds to an increase in suction at the upper plate and thus to an increase of the lateral mass flux out of the upper plate (e.g. trans-capillary membrane diffusion efflux) which will drag the fluid towards the upper plate, enhancing momentum in the y-direction and depleting momentum in the x-direction and, as a result, generating a significant deceleration of the blood velocity in the $\mathrm{x}$-direction. Figures 6 and 7 illustrate these conclusions for the case $H a=0$; for the case $H a=1$ (and for any other value of $\mathrm{Ha}$ on its nominal interval [0,2]) the figures look similar.

For fixed values of the Reynolds number we studied the influence of the Hartmann number on the velocities $U$ and $V$. As expected, since the magnetic field is applied in the y-direction, an increase of $\mathrm{Ha}$ do not have a visible influence on the $V(y)$ component of the blood velocity, leading to a decrease of the $U(y)$ component. This behavior is illustrated for $R e=1$ in Figures 8 and 9, and we mention that similar figures are obtained for any values of the Reynolds number on its nominal interval $[1,20]$. 


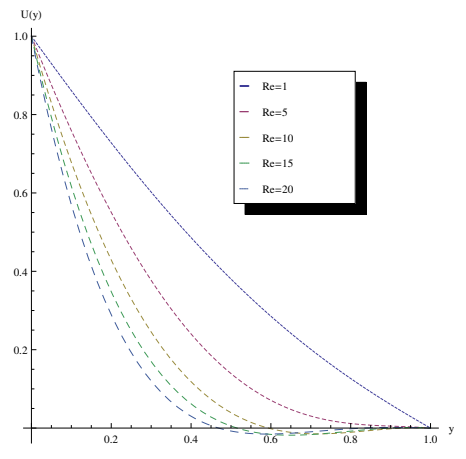

(a) 2D plot

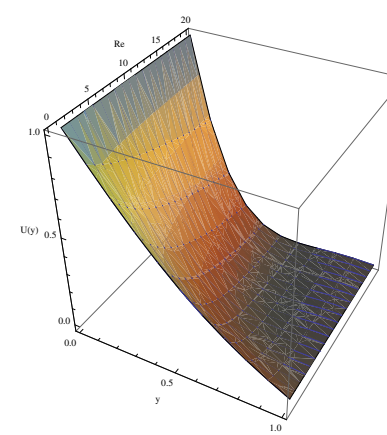

(b) 3D plot

Figure 6: The effect of the increase of $R e$ on $U$ for the case $H a=0$

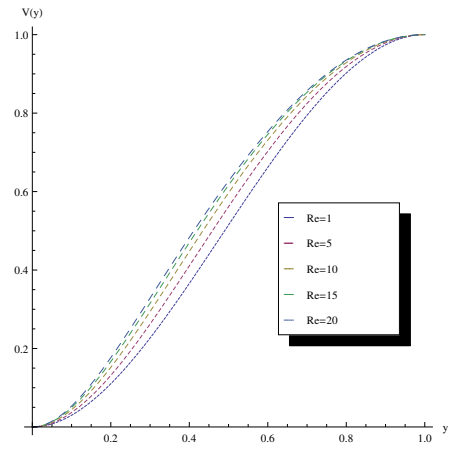

(a) $2 \mathrm{D}$ plot

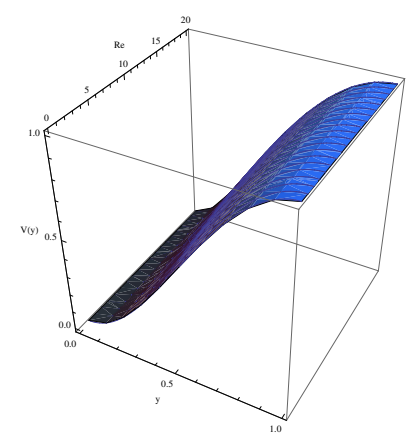

(b) $3 \mathrm{D}$ plot

Figure 7: The effect of the increase of $R e$ on $V$ for the case $H a=0$

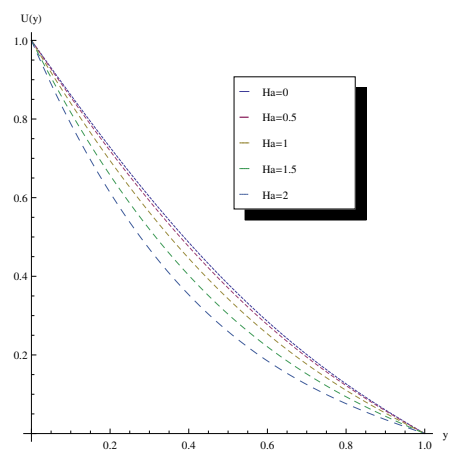

(a) $2 \mathrm{D}$ plot

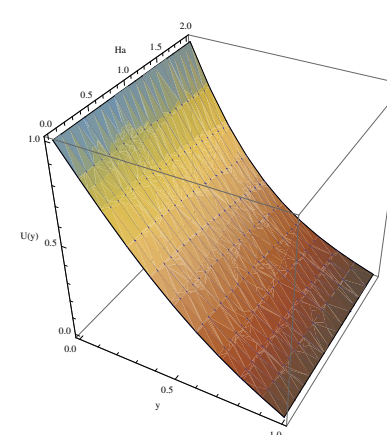

(b) $3 \mathrm{D}$ plot

Figure 8: The effect of the increase of $H a$ on $U$ for the case $R e=1$ 


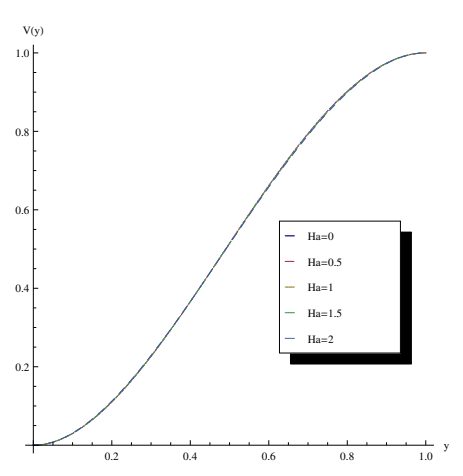

(a) 2D plot

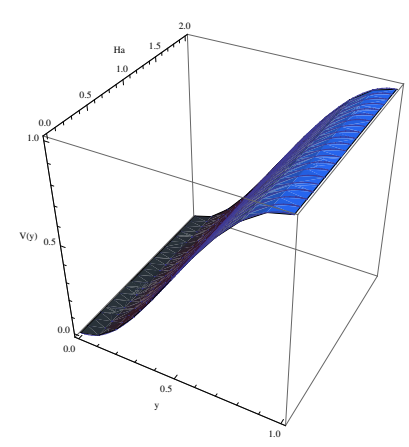

(b) 3D plot

Figure 9: The effect of the increase of $H a$ on $V$ for the case $R e=1$

Finally, we studied the combined influence of the Reynolds and Hartmann numbers on the $U(y)$ and $V(y)$ components of the blood velocity, combined influence which to our best knowledge, was not explicitly presented before. In order to illustrate graphically this combined influence, the surfaces in the Figures 10 and 11 represent the dependence on $R e$ and $H a$ of the values of $U(y)$ and $V(y)$, respectively, computed in $y=0.25$ (red surface, quarter of the channel), $y=0.5$ (green surface, middle of the channel) and $y=0.75$ (blue surface, three-quarters of the channel).

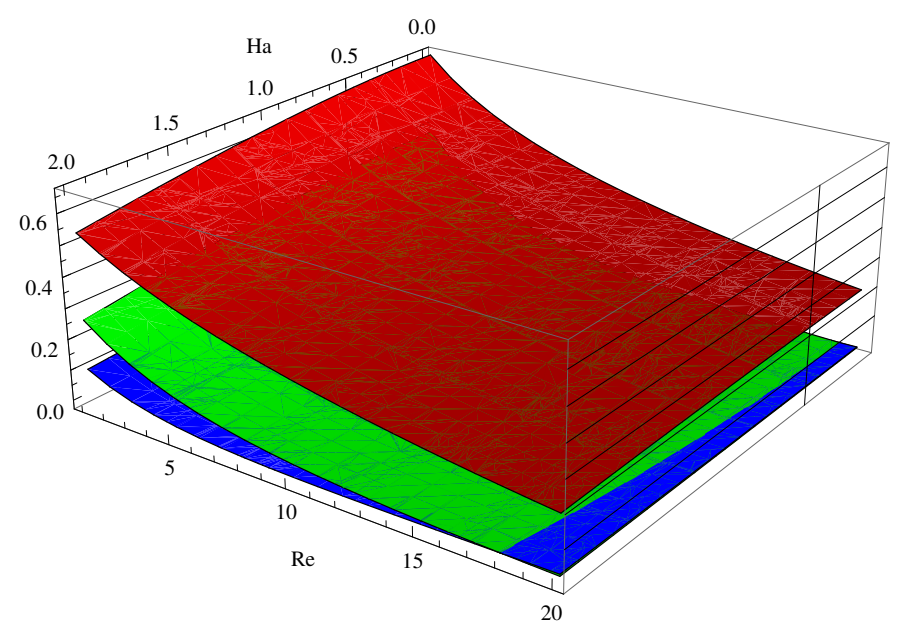

Figure 10: The combined influence of $R e$ and $H a$ on $U$. The red surface corresponds to $y=0.25$, the green surface to $y=0.5$ and the blue one to $y=0.75$

Figure 10 can be considered a good synthesis of the study of the influence of the Reynolds $\mathrm{Re}$ and Hartmann $\mathrm{Ha}$ numbers on the simulated blood flow in a semi-porous channel: as highlighted in the previous theoretical and numerical studies, the increase in both $\mathrm{Re}$ and $\mathrm{Ha}$ leads to a reduction of the blood flow velocity in the x-direction, $U$. 
Figure 10 also offers a new insight related to the interplay between the influence of $R e$ and that of $\mathrm{Ha}$ on $\mathrm{U}$ - namely the fact that the decelerative effect of the increase of $\mathrm{Ha}$ strongly depends on $R e$. For small values of $R e$ this effect is the most pronounced and, as a consequence, in situations where due to practical considerations a strong suction at the upper wall (characterized by a large value of $R e$ ) can not be obtained, a reduction of the blood flow velocity can be obtained by increasing the intensity of the magnetic field applied. However, when practically possible, an increase of the suction at the upper wall seems to be the preferable method of reduction of the flow, since the effect of the increase of $R e$ seems to be much stronger than the effect of the increase of $\mathrm{Ha}$. Moreover, for large values of $R e$, the effect of the increase of $\mathrm{Ha}$ is very small.

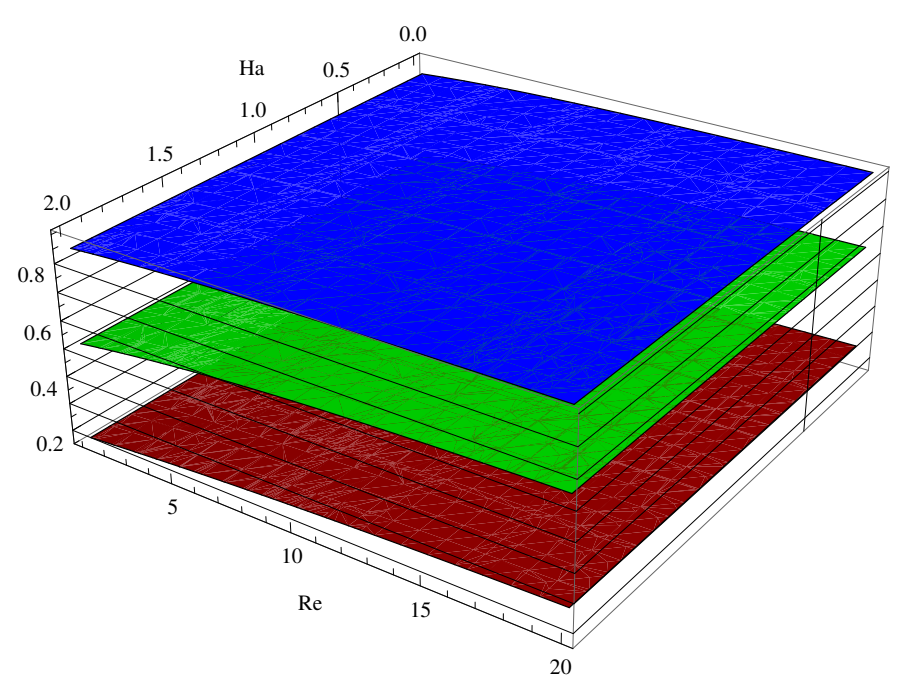

Figure 11: The combined influence of $R e$ and $H a$ on $V$. The red surface corresponds to $y=0.25$, the green surface to $y=0.5$ and the blue one to $y=0.75$

\section{Conclusions}

In this paper new and more accurate analytical approximate solutions for the magnetohemodynamic laminar viscous flow of a conducting physiological fluid in a semi-porous channel under a transverse magnetic field are computed by using the Polynomial Least Squares Method PLSM.

The comparison with previous approximate solutions computed by using DTM and HAM clearly illustrate the precision of the method, since the solutions computed by using PLSM are not only more accurate but also present a much simpler expression.

The analysis of the approximate solutions corresponding to various values of the Reynolds number Re and of the Hartmann number $\mathrm{Ha}$ presented in the last section can shed some light on the interplay of the influence of these parameters on the blood flow velocity and their possible contribution on the regularization and control of the blood flow. 


\section{References}

[1] V.P.Srivastava, A Theoretical Model for Blood Flow in Small Vessels, International Journal Of Application and Applied Mathematics Volume 2, 51-65 (2007).

[2] C.Y.Wang, J.B.Bassingthwaighte, Blood Flow in Small Curved Tubes, Journal of Biomechanical Engineering Volume 125, 910-913 (2003).

[3] R.Bali, U.Awasthi, Mathematical model of Blood Flow in Small Blood Vessel in the Presence of Magnetic Field, Applied mathematics Volume 2, 264-269 (2001).

[4] T.Yamamoto, Y.Nagayama, M.Tamura, A blood-oxygenation-dependent increase in blood viscosity due to a static magnetic field, Phys. Med. Biol. Volume 49, 3267-3267 (2004).

[5] A.Basiri Parsa, M.M.Rashidi, O.Anwar Beg, S.M.Sardi, Semi-computational simulation of magneto-hemodynamic flow in a semi-porous channel using optimal homotopy and differential transform methods, Computers in Biology and Medicine Volume 43, 1142-1153(2013).

[6] A.Alshare, B.Tashtoush, H.H.El-Khalil, Computational Modeling of Non-Newtonian Blood Flow Through Stenosed Arteries in the Presence of Magnetic Field, Journal of Biomechanical Engineering Volume 135, 114503 (6 pages) (2013).

[7] H.C.Weng, Hydrodynamic Modeling of Targeted Magnetic-Particle Delivery in a Blood Vessel, Journal of Biomechanical Engineering Volume 135, 034504 (5 pages) (2013).

[8] Kh.S.Mekheimer, Peristaltic flow of blood under effect of a magnetic field in a nonuniform channels, Applied Mathematics and Computation 153, 2004, 763-777.

[9] T.S.Tenforde, Magnetically induced electric fields and currents in the circulatory system, Progress in Biophysics and Molecular Biology Volume 87, 279-288 (2005).

[10] J.Ryu, X.Hu, S.C.Shadden, A Coupled Lumped-Parameter and Distributed Network Model for Cerebral Pulse-Wave Hemodynamics, Journal of Biomechanical Engineering Volume 137, 2015, 101009 (13 pages) (2015).

[11] D.Srinivasacharya, G.Madhava Rao, Mathematical model for blood flow through a bifurcated artery using couple stress fluid, Mathematical Biosciences Volume 278, 37-47 (2016).

[12] A.Sinha MHD flow and heat transfer of a third order fluid in a porous channel with stretching wall: Application to hemodynamics, Alexandria Engineering Journal Volume 54, 1243-1252 (2015).

[13] A.Zaman, N.Ali, M.Sajid, Numerical simulation of pulsatile flow of blood in a poroussaturated overlapping stenosed artery, Mathematics and Computers in Simulation, DOI: 10.1016/j.matcom.2016.09.008 (2016).

[14] A.S. Berman, Laminar flow in channels with porous walls, J. Appl. Phys. Volume 24, 1232-1235 (1953).

[15] F.M.Skalak, C.Y.Wang, On the non-unique solutions of laminar flow through a porous tube or channel, SIAM Journal on Applied Mathematics Volume 34, 535-544 (1978).

[16] J.P. Quaile, E.K. Levy, Laminar flow in a porous tube with suction, International Journal of Heat and Mass Transfer Volume 97, 223-243 (1975).

[17] E.A. Evans, R. Skalak, Mechanics and Thermodynamics of Biomembranes, CRC Press, Florida, USA, 1980. 